\section{Halbsättigungsdruck (Hämoglobin)}

O. Müller-Plathe

Hamburg, Deutschland

$\operatorname{Synonym(e)~} p 50$

Englischer Begriff partial pressure of oxygen at half saturation; $p 50 ; p \mathrm{O}_{2}(0,5)$

Definition $p 50$ ist derjenige Sauerstoffpartialdruck, bei dem das bindungsfähige Hämoglobin $(\mathrm{Hb})$ zur Hälfte mit $\mathrm{O}_{2}$ gesättigt ist, bei dem also $s \mathrm{O}_{2} 0,50$ beträgt.

Funktion - Pathophysiologie $p 50$ ist der quantitative Ausdruck für die $\mathrm{O}_{2}$-Affinität des Hämoglobins:

\begin{tabular}{ll|l|l}
$\mathrm{O}_{2}$-Affinität & $\mathrm{O}_{2}$-Dissoziationskurve & $p 50$ & $\mathrm{O}_{2}$-Abgabe \\
\hline$\uparrow$ & Links $\leftarrow$ & $\downarrow$ & $\downarrow$ \\
$\downarrow$ & $\rightarrow$ Rechts & $\uparrow$ & $\uparrow$
\end{tabular}

$\mathrm{Zu}$ den Ursachen einer veränderten $\mathrm{O}_{2}$-Affinität $>$ Sauerstofftransport. Die erheblichen Auswirkungen von Affinitätsänderungen auf die $\mathrm{O}_{2}$-Abgabe sind dort in der Abbildung am Beispiel des pH-Einflusses veranschaulicht.

Untersuchungsmaterial - Entnahmebedingungen Nach kräftiger Venenstauung von etwa 2 Minuten in einer Blutgasspritze abgenommenes Heparinblut $\left(s \mathrm{O}_{2} 0,40-0,80\right)$.

Probenstabilität $>$ Blutgasanalyse.

Präanalytik $>$ Blutgasanalyse.
Analytik Messung von $p \mathrm{O}_{2}, p \mathrm{CO}_{2}, \mathrm{pH}$ und $s \mathrm{O}_{2}$ mit dem Blutgasanalysator. Berechnung des aktuellen $p 50$ (bezogen auf die aktuellen Werte für $\mathrm{pH}$ und $p \mathrm{CO}_{2}$ ):

$$
\begin{gathered}
\lg p 50(\text { akt })=\lg p \mathrm{O}_{2}-\operatorname{logit} s \mathrm{O}_{2} / 2,7 \\
\operatorname{logit} s \mathrm{O}_{2}=\lg \left(s \mathrm{O}_{2} /\left[1-s \mathrm{O}_{2}\right]\right)
\end{gathered}
$$

Die Berechnung wird in größeren Blutgasanalysatoren automatisch durchgeführt.

\section{Konventionelle Einheit $\mathrm{mmHg}$.}

Internationale Einheit $\mathrm{kPa}$.

Umrechnungsfaktor zw. konv. u. int. Einheit 0,133 .

Referenzbereich - Erwachsene $25-29 \mathrm{mmHg}, 3,3-3,9 \mathrm{kPa}$.

Referenzbereich - Kinder 25-29 mmHg, 3,3-3,9 kPa; Neugeborene: $18-24 \mathrm{mmHg}, 2,4-3,2 \mathrm{kPa}$ (niedriger durch Anteile von fetalem $\mathrm{Hb}$ ).

Indikation $>$ Sauerstofftransport.

Interpretation Der aktuelle $p 50$ gibt Auskunft über die momentane $\mathrm{O}_{2}$-Affinität unter Berücksichtigung aller Einflüsse und ist bei kritischer $\mathrm{O}_{2}$-Versorgung in der Akutmedizin von Bedeutung.

\section{Literatur}

International Federation of Clinical Chemistry (1990) Guidelines for routine measurement of blood oxygen affinity. Scand J Lab Invest 50(Suppl. 203):227-234 